\title{
Bimodal distribution of blocking temperature in exchange-biased ferromagnetic/antiferromagnetic bilayers
}

\author{
V. Baltz, ${ }^{1, *}$ B. Rodmacq, ${ }^{1}$ A. Zarefy, ${ }^{2}$ L. Lechevallier, ${ }^{2}$ and B. Dieny ${ }^{1}$ \\ ${ }^{1}$ SPINTEC, UMR 8191 CEA/CNRS/UJF/Grenoble INP, INAC, 17 rue des Martyrs, 38054 Grenoble Cedex, France \\ ${ }^{2}$ GPM, UMR 6634 CNRS-Université de Rouen, Avenue de l'Université, BP 12, 76801 St Etienne du Rouvray, France
}

(Received 6 October 2009; published 10 February 2010)

\begin{abstract}
The blocking temperature $\left(T_{\mathrm{B}}\right)$ distribution of various polycrystalline ferromagnetic $(\mathrm{F}) /$ antiferromagnetic (AF) bilayers exhibits two distinct peaks. This is ascribed to the existence of two families of magnetic entities determining the temperature dependence of exchange bias. One is formed by the AF grains which undergo thermally activated magnetic reversal. The other is formed by F/AF disordered interfacial spins which exhibit spin-glass-like behavior. The bulk versus interfacial nature of these two families is indicated by the dependence of the $T_{\mathrm{B}}$ distribution on the AF thickness.
\end{abstract}

DOI: 10.1103/PhysRevB.81.052404

PACS number(s): 75.70.Cn, 75.30.Et, 75.60.Nt, 75.75.-c

Nowadays, exchange bias (EB) (Ref. 1) is used in a large variety of spintronic devices. ${ }^{2}$ It allows pinning the magnetization of a ferromagnetic layer in a fixed direction thus defining a reference direction for the spin of conduction electrons. It is named after the hysteresis loop shift (bias) along the magnetic field axis, which results from the exchange interaction between a ferromagnet $(\mathrm{F})$ and an antiferromagnet $(\mathrm{AF})$ in direct contact. Most often, EB is used with AF polycrystalline alloys (IrMn, PtMn, FeMn, and NiMn). It was already shown that in such systems, EB results for the combination of several phenomena. The AF layer is viewed as an assembly of almost uncoupled grains having a pinning energy $K V$, where $K$ is the AF anisotropy energy per unit volume and $V$ the grain volume. The latter usually follows a lognormal distribution $P(V)$. The AF spin lattice within each $\mathrm{AF}$ grain is coupled to the magnetization of the adjacent $\mathrm{F}$ layer by an effective interfacial exchange interaction per unit area $J_{\mathrm{F} / \mathrm{AF} \cdot}{ }^{1,3-5}$ In EB experiments, the $\mathrm{AF}$ spin-lattice orientation is first set by field cooling the F/AF bilayer under a magnetic field from above the AF Néel temperature. The external field polarizes the $\mathrm{F}$ magnetization so that the $\mathrm{AF}$ spin lattice adopts a magnetic ordering during cooling which satisfies as much as possible the F/AF interfacial exchange interactions. Once back to low temperature, if a large enough reverse field is applied to switch the F magnetization, then the AF spin lattice within each grain is submitted to a torque per unit area given by $J_{\mathrm{F} / \mathrm{AF}}{ }^{1,6-8}$ Depending on the pinning energy $K V$ and thermal activation $\log \left(\tau / \tau_{0}\right) k_{B} T$, the $\mathrm{AF}$ grain spin lattice can resist or not to this torque $(\tau$ is the characteristic time of the experiment $\sim 1 \mathrm{~s}, \tau_{0}$ the attempt time $\sim 10^{-9} \mathrm{~s}, k_{B}$ the Boltzmann constant, $T$ the temperature). By taking into account the above energies, we can therefore write the criterion for stability of the spin lattice of a given $\mathrm{AF}$ grain in the following form: ${ }^{1}$

$$
\left[K-\frac{J_{\mathrm{F} / \mathrm{AF}}}{t_{\mathrm{AF}}}\right] V>\log \left(\tau / \tau_{0}\right) k_{B} T,
$$

$t_{\mathrm{AF}}$ being the $\mathrm{AF}$ layer thickness. The blocking temperature $\left(T_{\mathrm{B}}\right)$ of an $\mathrm{AF}$ grain is defined by the corresponding equality. The large enough stable AF grains contribute to the EB loop shift of the F layer whereas the switching ones contribute to the F/AF bilayer coercivity. ${ }^{1}$ The EB loop shift $H_{\mathrm{E}}$ can then be expressed like,

$$
M_{S} H_{\mathrm{E}} t_{\mathrm{F}}=\int_{\text {stable grains }} J_{\mathrm{F} / \mathrm{AF}} \frac{V}{t_{\mathrm{AF}}} P(V) d V,
$$

where $M_{S}$ and $t_{\mathrm{F}}$ are the saturation magnetization and the thickness of the $\mathrm{F}$ layer, respectively. Besides this simple picture, the temperature dependence of EB is complicated by several factors: (i) the anisotropy $K$ of the AF layer is temperature dependent. It is often assumed that it varies as the third power of the AF sublattice magnetization; ${ }^{4}$ (ii) the interfacial coupling may strongly depend on temperature. It was very early pointed out that, due to interfacial roughness, spatially random positive or negative coupling within a grain may exist through $\mathrm{F} / \mathrm{AF}$ interfaces resulting in a high degree of interfacial exchange frustration as in random field systems ${ }^{9}$ or spin glasses. ${ }^{10}$ Due to these competing interactions, interfacial spins may locally be highly disordered resulting in a quite reduced molecular field. ${ }^{9-12}$ This disorder surely evolves dynamically during hysteresis loops. Wherever the frustration is particularly high, the interfacial spins behave like a spin glass below the freezing temperature $T_{\text {freezing }}$ and as a spin liquid above $T_{\text {freezing }}$, the latter being only a fraction of the AF Néel temperature. ${ }^{10}$ These spinglass regions induce a strong effective $T$ variation in $J_{\mathrm{F} / \mathrm{AF}}$ since $\mathrm{F}$ and $\mathrm{AF}$ are essentially decoupled above $T_{\text {freezing }}$ and couple below $T_{\text {freezing. }}$. In contrast, within a given grain, in interfacial regions where the frustration is weak, $\mathrm{F}$ and $\mathrm{AF}$ are magnetically coupled up to $T_{\mathrm{B}}$. A gradual freezing of the interfacial frustrated spins at low $T$ has been proposed as an explanation for the low- $T$ sharp increase in the thermal variation in EB field, as observed in several F/AF bilayers. ${ }^{5,13-15}$ We believe that as a result of the spatial fluctuations in interfacial roughness and correlatively in frustration of exchange interactions across the F/AF interface, a distribution of $J_{\mathrm{F} / \mathrm{AF}}$ interfacial coupling also exists, which we write $D\left(J_{\mathrm{F} / \mathrm{AF}}\right)$. This other factor also contributes to the thermal dependence of exchange bias. Taking into account this temperature-dependent distribution $D\left(J_{\mathrm{F} / \mathrm{AF}}\right)$, Eq. (2) then writes, 

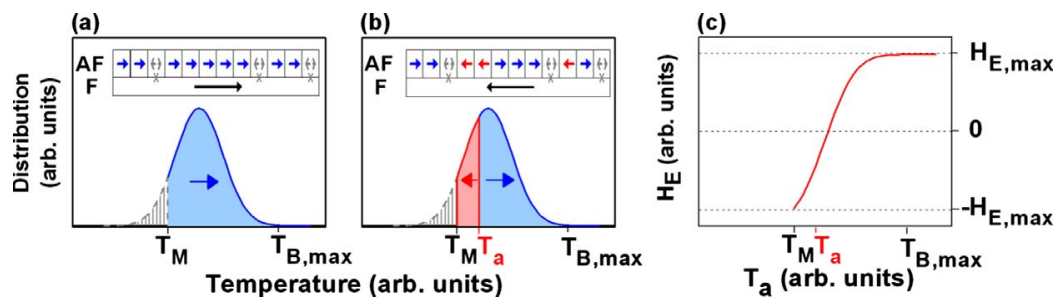

FIG. 1. (Color online) (a) Sketch of the orientation of the AF entities along with visualization of the blocking temperature ( $T_{\mathrm{B}}$ ) distribution after: (i) FC under a positive field from above the maximum $T_{\mathrm{B}}\left(T_{\mathrm{B} \text {,max }}\right)$ down to the temperature of measurement $\left(T_{\mathrm{M}}\right)$. (b) Same representation after: step (i) and (ii) FC under a negative field from an annealing temperature $\left(T_{\mathrm{a}}\right)$ down to $T_{\mathrm{M}}$. (c) Sketch of the dependence of the EB field $\left(H_{\mathrm{E}}\right)$ measured at $T_{\mathrm{M}}$ as a function of $T_{\mathrm{a}}$.

$$
M_{S} H_{\mathrm{E}} t_{\mathrm{F}}=\iint_{\text {stable grains }} J_{\mathrm{F} / \mathrm{AF}} D\left(J_{\mathrm{F} / \mathrm{AF}}\right) \frac{V}{t_{\mathrm{AF}}} P(V) d V d J_{\mathrm{F} / \mathrm{AF}} .
$$

In this Brief Report, we evidenced bimodal distributions of blocking temperatures. More precisely, we established the presence of an additional contribution to the thermal variation in exchange bias resulting from a temperature-dependent distribution of coupling across the interface. We experimentally confirmed that two families of AF entities contribute to the $T$ dependence of EB: the AF grains themselves which are submitted to thermally activated magnetic reversal and the spin-glass-like interfacial region which influences the strength of the short-distance coupling across the F/AF interface. The bulk versus interfacial character of these two families is derived from the dependence of the $T_{\mathrm{B}}$ distribution on $t_{\mathrm{AF}}$.

The samples were prepared by dc sputtering on $\mathrm{Si} / \mathrm{SiO}_{2}$ substrates in a $2.5 \times 10^{-3}$ mbar Ar atmosphere with deposition rates between 0.05 and $0.1 \mathrm{~nm} \mathrm{~s}^{-1}$. 16,17 The films compositions are (from bottom to top) NiFe (4 nm)/FeMn $(9 \mathrm{~nm})$ and $\mathrm{NiFe}(4 \mathrm{~nm}) / \mathrm{NiMn}(50 \mathrm{~nm})$, deposited on a Ta $(5 \mathrm{~nm})$ buffer, and Co $(3 \mathrm{~nm}) / \mathrm{IrMn}\left(t_{\mathrm{IrMn}}\right)$ deposited on a Ta (3 $\mathrm{nm}) / \mathrm{Cu}(3 \mathrm{~nm})$ buffer. The IrMn thickness takes values of 3 , 4 , and $7 \mathrm{~nm}$. All samples are capped in order to protect them from oxidation, either with a Ta $(5 \mathrm{~nm})$ layer or a Pt $(3 \mathrm{~nm})$ layer. $\mathrm{NiFe}$ was sputtered from a $\mathrm{Ni}_{81} \mathrm{Fe}_{19}$ target and antiferromagnetic FeMn, NiMn, and IrMn materials were deposited from $\mathrm{Fe}_{50} \mathrm{Mn}_{50}, \mathrm{Ni}_{50} \mathrm{Mn}_{50}$, and $\mathrm{Ir}_{20} \mathrm{Mn}_{80}$ targets, respectively.

The initial EB was set by postannealing and field cooling (FC) the samples down to $300 \mathrm{~K}$ under a $5 \times 10^{-6}$ mbar vacuum, from above their maximum blocking temperature $\left(T_{\mathrm{B}, \max }\right)$ in an in-plane magnetic field $\left(H_{\mathrm{FC}}=2.4 \mathrm{kOe}\right)$ large enough to saturate the magnetization of the $\mathrm{F}$ layer. The FC started from $580 \mathrm{~K}$ for $\mathrm{NiFe} / \mathrm{NiMn}$ and from $500 \mathrm{~K}$ for the $\mathrm{NiFe} / \mathrm{FeMn}$ - and $\mathrm{Co} / \mathrm{IrMn}$-based samples, these initial temperatures being above $T_{\mathrm{B} \text {,max }}$ at the time scale of the experiment. ${ }^{17,18}$

Magnetic hysteresis loops were measured by superconducting quantum interference device (SQUID) magnetometry. In order to determine the blocking temperature $\left(T_{\mathrm{B}}\right)$ distribution, measurements were taken at a fixed measuring temperature $\left(T_{\mathrm{M}}\right)$, chosen equal to $4 \mathrm{~K}$, following a specific field cooling procedure explained in Refs. 17 and 19. The procedure consists in successive steps. (i) The samples are first FC from above $T_{\mathrm{B} \text {, max }}$ down to $T_{\mathrm{M}}$ under a positive field.
At this point, all the AF entities whose blocking temperature are comprised between $T_{\mathrm{B} \text {, max }}$ and $T_{\mathrm{M}}$, are magnetically polarized toward the field direction (positive here) [Fig. 1(a)]. (ii) The temperature is then increased to an intermediate annealing temperature $\left(T_{\mathrm{a}}\right.$, lower than $\left.T_{\mathrm{B}, \max }\right)$. Subsequently, the samples are FC under a negative field, down to $T_{\mathrm{M}}$. At this step, the AF entities whose $T_{\mathrm{B}}$ is comprised between $T_{\mathrm{a}}$ and $T_{\mathrm{M}}$ have been magnetically repolarized toward the negative direction [Fig. 1(b)]. By repeating these two steps for increasing $T_{\mathrm{a}}$ the $\mathrm{AF}$ entities are progressively reoriented, thus describing the $T_{\mathrm{B}}$ distribution. Then by plotting the EB field $\left(H_{\mathrm{E}}\right)$ as measured at $T_{\mathrm{M}}$ as a function of $T_{\mathrm{a}}$ [Fig. 1(c)], we get the integrand of the $T_{\mathrm{B}}$ distribution. Indeed, $H_{\mathrm{E}}$ at each increment of $T_{\mathrm{a}}$ is proportional to the difference between the amount of entities oriented positively and negatively. ${ }^{16-19}$ We note that the AF spins for which $T_{\mathrm{B}}$ is lower than $T_{\mathrm{M}}$ do not contribute to $H_{\mathrm{E}}$ at $T_{\mathrm{M}}$ since thermal activation overcomes the anisotropy and F/AF interfacial exchange energy in these regions. As a consequence, below $T_{\mathrm{M}}$, no information on the $T_{\mathrm{B}}$ distribution can be derived. It is also important to note that here, in contrast to $H_{\mathrm{E}}$ vs $T$ measurements, all the loops are made at a fixed temperature. This allows us to get rid of the temperature variations in the physical parameters and thereby to derive information directly on the distributions of parameters.

The procedure discussed in the above paragraph has been applied to the Co $(3 \mathrm{~nm}) / \mathrm{IrMn}\left(t_{\mathrm{IrMn}}\right)$ films. Typical hysteresis loops measured at $4 \mathrm{~K}$ and after various $T_{\mathrm{a}}$ are shown in Fig. 2, for $t_{\mathrm{IrMn}}=7 \mathrm{~nm}$. The evolutions of $H_{\mathrm{C}}$ and $H_{\mathrm{E}}$ with $T_{\mathrm{a}}$ for $t_{\mathrm{IrMn}}=3,4$, and $7 \mathrm{~nm}$ are plotted in Figs. 3(a) and 3(b), respectively. $H_{\mathrm{E}}$ and $H_{\mathrm{C}}$ are deduced from the sets of hysteresis loops and are, respectively, defined as the offset along the field axis and the half width of the loop.

From Fig. 3(a), we observe that $H_{\mathrm{C}}$ remains almost independent of $T_{\mathrm{a}}$ for all $t_{\mathrm{IrMn}} \cdot H_{\mathrm{C}}$ is primarily related to the AF grains whose spin lattice is dragged during the magnetization reversal of the $\mathrm{F}$ layer. These grains are those who do not satisfy inequality (1). At $T_{\mathrm{M}}$, this inequality is independent on the cooling history between $T_{\mathrm{a}}$ and $T_{\mathrm{M}}$ which explains the independence of $H_{\mathrm{c}}$ on $T_{\mathrm{a}}$. For all samples, Fig. 3(b) shows that $H_{\mathrm{E}}$ increases with increasing $T_{\mathrm{a}}$ and changes sign, as explained above (Fig. 1). However, striking differences in the shape of the dependences of $H_{\mathrm{E}}$ on $T_{\mathrm{a}}$ are noticeable depending on $t_{\mathrm{IrMn}}$ : for $t_{\mathrm{IrMn}}=3 \mathrm{~nm}, H_{\mathrm{E}}\left(T_{\mathrm{a}}\right)$ exhibits a negative curvature and levels off for high $T_{\mathrm{a}}$, which contrasts with the two other samples for which an inflection point in $H_{\mathrm{E}}\left(T_{\mathrm{a}}\right)$ is observed. These features yield significant differences in 


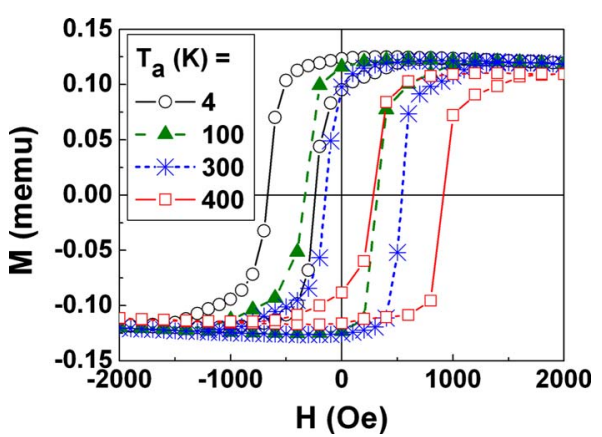

FIG. 2. (Color online) Hysteresis loops for films of Ta (3 $\mathrm{nm}) / \mathrm{Cu}(3 \mathrm{~nm}) / \mathrm{Co}(3 \mathrm{~nm}) / \mathrm{IrMn}(7 \mathrm{~nm}) / \mathrm{Pt}(2 \mathrm{~nm})$, for different annealing temperatures $\left(T_{\mathrm{a}}\right)$. The samples were initially FC under a positive saturating field of $10 \mathrm{kOe}$ from above $T_{\mathrm{B}, \max }$ down to $4 \mathrm{~K}$. They were then FC under $-10 \mathrm{kOe}$ from $T_{\mathrm{a}}$ down to $4 \mathrm{~K}$. The loops were recorded at $4 \mathrm{~K}$ by SQUID along the FC direction.

the derivatives of $H_{\mathrm{E}}$ vs $T_{\mathrm{a}}\left(\delta H_{\mathrm{E}} / \delta T_{\mathrm{a}}\right)$, which represent the $T_{\mathrm{B}}$ distributions. ${ }^{17,19}$ They are plotted in Fig. 3(c). These distributions account for the amount of AF entities, which are "pinned" below $T_{\mathrm{a}}$ and start being dragged at $T=T_{\mathrm{a}}{ }^{1,16-19}$ Two peaks in the $T_{\mathrm{B}}$ distribution can be distinguished for $t_{\mathrm{IrMn}}=4$ and $7 \mathrm{~nm}$, which is unusual. The wide distribution for $t_{\mathrm{IrMn}}=3 \mathrm{~nm}$ suggests that the two peaks are overlapping. The high- $T$ peak shifts toward higher $T$ as $t_{\mathrm{IrMn}}$ increases (Fig. 3). This particular peak is therefore related to the bulk part of the $\mathrm{AF}$, i.e., to the $\mathrm{AF}$ grains themselves which spin lattice either remains pinned or is dragged during the F magnetization switching. It is the commonly observed peak and trend, ${ }^{1,16-19}$ which consider that the magnetization reversal of the $\mathrm{F}$ results in a dynamical reorientation of the AF, not only at its F/AF interface, but also in its bulk. ${ }^{1,3,5,7,8}$ This high- $T$ peak in the $T_{\mathrm{B}}$ distribution is thus directly related to the grain size distribution $P(V)$ of the above equality (3). In the literature, the measurement temperature of $300 \mathrm{~K}$ has been so far chosen as $T_{\mathrm{M}}$. It allowed the observation of this well-known $T_{\mathrm{B}}$ distribution, which exhibits a single high- $T$ peak. It is often studied in view of understanding the influences of the structural properties and magnetic parameters (e.g., anisotropy, exchange stiffness) of the F/AF bilayers on the mean value, maximum value, or standard deviation of the distribution. ${ }^{1,16-19}$ The main result we present here is the direct observation of an unusual second peak in the $T_{\mathrm{B}}$ distribution. In contrast to the well-known high- $T$ peak, this low- $T$ contribution to the $T_{\mathrm{B}}$ distribution looks independent on $t_{\mathrm{IrMn}}$. Because of this independence on $t_{\mathrm{IrMn}}$, the magnetic entities associated with this second low- $T$ contribution are likely associated with the F/AF interfacial spin-glass-like region exhibiting low freezing temperature (typically between 4 and $70 \mathrm{~K}$ ). These regions undergo spin reorganization when the sample is annealed at $T_{\mathrm{a}}$ typically between 4 and $70 \mathrm{~K}$ and FC in opposite field. These interfacial spin reorganization locally change the effective coupling $J_{\mathrm{F} / \mathrm{AF}}$ across these regions thus affecting the EB field [equality (3)]. These spinglass-like regions are probably those located nearby any particular defects, e.g., grain boundaries or roughness-induced steps, which are able to locally reduce the molecular field. The distribution of the above properties results in a distribution of $J_{\mathrm{F} / \mathrm{AF}}: D\left(J_{\mathrm{F} / \mathrm{AF}}\right)$, which is here directly probed [equality (3)]. Within a grain F and AF are magnetically coupled across spin-glass-like interfacial regions only up to $T_{\text {freezing, }}$, even if the AF core below may remain frozen up to $T_{\mathrm{B}}$. Over the whole sample, the resulting low- $T$ blocking temperature distribution is related to the distribution of $T_{\text {freezing }}$ which transposes in an effective distribution of $J_{\mathrm{F} / \mathrm{AF}}: D\left(J_{\mathrm{F} / \mathrm{AF}}\right)$. In contrast, within a grain, in interfacial regions where the frustration is weak, $\mathrm{F}$ and $\mathrm{AF}$ are magnetically coupled up to $T_{\mathrm{B}}$.

We note that some insights on the possible coexistence of different categories of magnetic entities in F/AF bilayers, influencing $T_{\mathrm{B}}$ distributions, have been disseminated in the literature. ${ }^{5,13-15,20}$ In Ref. 13, for example, correlated experiments on $\mathrm{CoO} / \mathrm{MgO}$ and $\mathrm{NiFe} / \mathrm{CoO}$ multilayers allowed the authors to indirectly observe manifestations of a $T_{\mathrm{B}}$ distribution exhibiting two peaks. Despite the fundamental importance of this finding, direct proofs of the presence of multipeaks in the $T_{\mathrm{B}}$ distributions and of their origin was lacking. In Ref. 5, a gradual freezing of the interfacial frustrated spins over the whole range of temperature has been proposed, with an interfacial coupling varying like $\left(1-T / T_{0}\right)^{2 / 3}$. Such a smooth variation however leads to a single-peaked $T_{\mathrm{B}}$ distribution, as witnessed by the black line in Fig. 7 of Ref. 5 and thus seems to fail in reproducing the low- $T$ sharp increase in the thermal variation in EB field. We rather believe that the distribution of $J_{\mathrm{F} / \mathrm{AF}}$ and the low-temperature freezing of
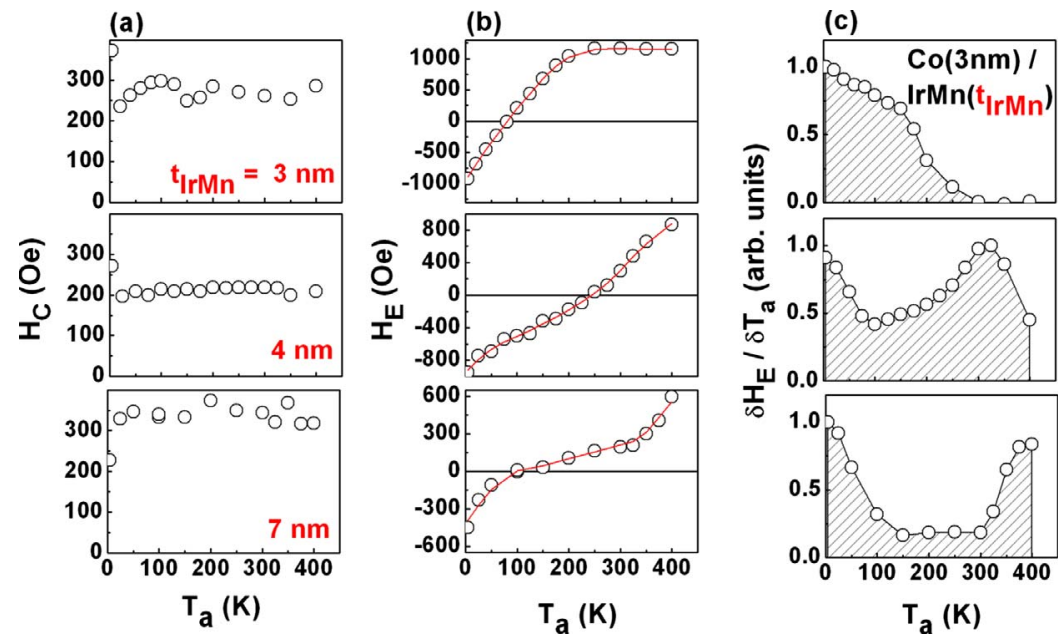

FIG. 3. (Color online) Dependence of (a) the coercive field $\left(H_{\mathrm{C}}\right)$, (b) EB field $\left(H_{\mathrm{E}}\right)$, and (c) derivative $\delta H_{\mathrm{E}} / \delta T_{\mathrm{a}}$ on the annealing temperature $\left(T_{\mathrm{a}}\right)$ for Co $(3 \mathrm{~nm}) / \operatorname{IrMn}\left(t_{\mathrm{IrMn}}=3,4\right.$, and $\left.7 \mathrm{~nm}\right)$ films. $\delta H_{\mathrm{E}} / \delta T_{\mathrm{a}}$ vs $T_{\mathrm{a}}$ are the blocking temperature distributions. $H_{\mathrm{C}}$ and $H_{\mathrm{E}}$ are determined after hysteresis loops subsequent to cooling procedures described in the caption of Fig. 2. The lines in (b) result from smoothing of the raw data and were used for the derivative. 

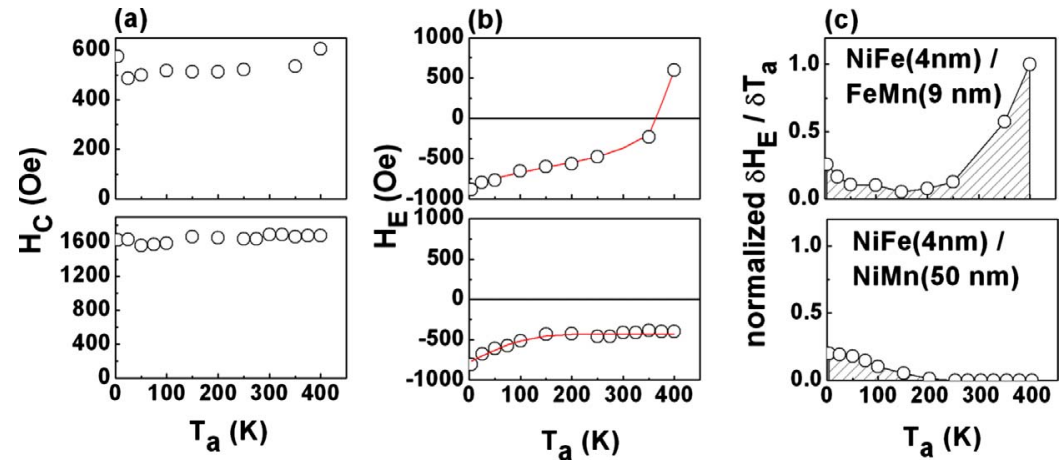

FIG. 4. (Color online) Dependence of (a) the coercive field $\left(H_{\mathrm{C}}\right),(\mathrm{b}) \mathrm{EB}$ field $\left(H_{\mathrm{E}}\right)$, and $(\mathrm{c})$ derivative $\delta H_{\mathrm{E}} / \delta T_{\mathrm{a}}$ on the annealing temperature $\left(T_{\mathrm{a}}\right)$ for $\mathrm{NiFe}(4 \mathrm{~nm}) / \mathrm{FeMn}(9 \mathrm{~nm})$ and $\mathrm{NiFe}(4$ $\mathrm{nm}) / \mathrm{NiMn}(50 \mathrm{~nm})$ films. these interfacial spin-glass regions are a clue to explain the large increase in exchange bias at low temperature.

From Fig. 3, we also observe that at $4 \mathrm{~K}$ (i.e., for $T_{\mathrm{a}}$ $=4 \mathrm{~K}$, after the first $\mathrm{FC}$ from above the maximum $T_{\mathrm{B}}$ ), the thinner the IrMn, the larger the amplitude of $H_{\mathrm{E}}$. We believe this is due to the fact that a thin antiferromagnetic layer acquires a higher overall polarization when in contact with a ferromagnet than a thicker AF layer. This means that at low temperature, a larger net moment of pinned uncompensated spins are present in the thin AF layer yielding a larger exchange-bias field as observed. Of course, if the measurement temperature is increased, the thin $\mathrm{AF}$ layer is more prone to thermally activated reversal so that the exchange bias more rapidly decreases than a thicker AF layer.

Figure 3(c) shows that the two peaks in the $T_{\mathrm{B}}$ distribution seem to have comparable amplitudes. This implies that when $T_{\mathrm{a}}$ is varied, the overall influence on $H_{\mathrm{E}}$ of the interfacial spin-glass-like region is comparable to the overall influence of the pinning/depinning of the AF grains. We attribute this to the highly disordered character of $\mathrm{Co} / \mathrm{IrMn}$ interface due to $\mathrm{Mn}$ diffusion, as evidenced in previous studies. ${ }^{21}$ This $\mathrm{Mn}$ interdiffusion may reinforce the spin-glass character of the $\mathrm{Co} / \mathrm{IrMn}$ interface.

Using the same experimental procedure, the $T_{\mathrm{a}}$ dependences of $H_{\mathrm{C}}, H_{\mathrm{E}}$, and $\delta H_{\mathrm{E}} / \delta T_{\mathrm{a}}$ for other F/AF bilayers: $\mathrm{NiFe}(4 \mathrm{~nm}) / \mathrm{FeMn}(9 \mathrm{~nm})$ and $\mathrm{NiFe}(4 \mathrm{~nm}) / \mathrm{NiMn}(50 \mathrm{~nm})$ samples were measured and are plotted in Figs. 4(a)-4(c).
For these systems, we again evidence a low- $T$ contribution to the $T_{\mathrm{B}}$ distribution [Fig. 4(c)]. For the NiFe/FeMn stack, we can also observe the usual peak at higher $T$. This is not the case for the $\mathrm{NiFe} / \mathrm{NiMn}$ stack, since this peak is known to occur around $550 \mathrm{~K}$, well above our maximum measurement annealing temperature of $400 \mathrm{~K}$. This explains the relatively modest increase in $H_{\mathrm{E}}$ with $T_{\mathrm{a}}$ in Fig. 4(b). For these two F/AF bilayers, we note that the amplitude of the low- $T$ peak is much smaller than that of the high- $T$ one, in contrast to the $\mathrm{Co} / \operatorname{IrMn}\left(t_{\mathrm{IrMn}}\right)$ system. This smaller amplitude of the low- $T$ peak probably means that the corresponding F/AF interfaces are less frustrated. It is possibly related to a lesser Mn diffusion resulting from a lower $\mathrm{Mn}$ concentration in the $\mathrm{Fe}_{50} \mathrm{Mn}_{50}$ and $\mathrm{Ni}_{50} \mathrm{Mn}_{50}$ alloys as compared to $\mathrm{Ir}_{20} \mathrm{Mn}_{80} \cdot{ }^{21}$

In conclusion, for various F/AF bilayers, we evidenced the existence of a bimodal distribution of magnetic entities responsible for $\mathrm{EB}$. For some specific AF thicknesses, we unambiguously observe two distinct peaks in the $T_{\mathrm{B}}$ distribution. The low-temperature peak is independent on the thickness of the antiferromagnet. It is ascribed to interfacial spin-glass-like region having freezing temperature in the range $4-70 \mathrm{~K}$. In contrast, the high-temperature peak shifts toward higher temperature when the thickness of the antiferromagnet is increased. This peak is ascribed to the AF grains themselves.

The authors are grateful to CROCUS Technology for kindly providing the FeMn and NiMn based samples. *vincent.baltz@cea.fr

${ }^{1}$ J. Nogués and I. K. Schuller, J. Magn. Magn. Mater. 192, 203 (1999); A. E. Berkowitz and K. Takano, ibid. 200, 552 (1999); R. L. Stamps, J. Phys. D 33, R247 (2000); M. Kiwi, J. Magn. Magn. Mater. 234, 584 (2001).

${ }^{2}$ B. Dieny, V. S. Speriosu, S. S. P. Parkin, B. A. Gurney, D. R. Wilhoit, and D. Mauri, Phys. Rev. B 43, 1297 (1991).

${ }^{3}$ E. Fulcomer and S. H. Charap, J. Appl. Phys. 43, 4190 (1972).

${ }^{4}$ M. D. Stiles and R. D. McMichael, Phys. Rev. B 60, 12950 (1999).

${ }^{5}$ L. E. Fernandez-Outon et al., J. Appl. Phys. 104, 093907 (2008).

${ }^{6}$ W. H. Meiklejohn and C. P. Bean, Phys. Rev. 102, 1413 (1956).

${ }^{7}$ L. Néel, Ann. Phys. (Paris) 2, 61 (1967).

${ }^{8}$ D. Mauri, H. C. Siegmann, P. S. Bagus, and E. Kay, J. Appl. Phys. 62, 3047 (1987).

\footnotetext{
${ }^{9}$ A. P. Malozemoff, Phys. Rev. B 35, 3679 (1987).

${ }^{10}$ M. Ali et al., Nature Mater. 6, 70 (2007).

${ }^{11}$ U. Nowak et al., Phys. Rev. B 66, 014430 (2002).

${ }^{12}$ F. Ernult et al., J. Appl. Phys. 94, 6678 (2003).

${ }^{13}$ K. Takano et al., Phys. Rev. Lett. 79, 1130 (1997).

${ }^{14}$ J. Ventura et al., J. Appl. Phys. 101, 113901 (2007).

${ }^{15}$ S.-F. Cheng and P. Lubitz, J. Appl. Phys. 87, 4927 (2000).

${ }^{16}$ V. Baltz et al., Phys. Rev. Lett. 94, 117201 (2005).

${ }^{17}$ V. Baltz et al., Phys. Rev. B 72, 104419 (2005).

${ }^{18}$ J.-P. Nozières et al., J. Appl. Phys. 87, 3920 (2000).

${ }^{19}$ S. Soeya, T. Imagawa, S. Mitsuoka, and S. Narishige, J. Appl. Phys. 76, 5356 (1994).

${ }^{20}$ J. D. Dutson et al., J. Phys. D 40, 1293 (2007).

${ }^{21}$ L. Lechevallier et al., Phys. Rev. B 79, 174434 (2009).
} 